\title{
Single-Pulse and Unidirectional Electrical Activation of the Cervical Vagus Nerve Reduces Tumor Necrosis Factor in Endotoxemia
}

\author{
Peder S Olofsson, ${ }^{1,2^{*}}$ Yaakov A Levine, ${ }^{3 *}$ April Caravaca, ${ }^{3}$ Sangeeta S Chavan, ${ }^{1}$ Valentin A Pavlov, ${ }^{1}$ \\ Michael Faltys, ${ }^{3}$ and Kevin J Tracey ${ }^{1,4}$ \\ ${ }^{1}$ Department of Biomedical Sciences, The Feinstein Institute for Medical Research, Manhasset, New York, United States of America; \\ ${ }^{2}$ Center for Molecular Medicine, Department of Medicine, Solna, Karolinska Institutet, Stockholm; ${ }^{3}$ SetPoint Medical Corporation, \\ Valencia, California, United States of America; and ${ }^{4}$ Department of Women's and Children's Health, Karolinska University Hospital, \\ Karolinska Institutet, Stockholm, Sweden
}

\begin{abstract}
The inflammatory reflex is a neural circuit defined by action potentials transmitted in the vagus nerve that regulate cytokine production in the spleen. Detailed mechanistic studies implicate the vagus nerve, the splenic nerve, a T-cell subset that produces acetylcholine under the control of adrenergic signals, and alpha7 nicotinic acetylcholine receptors ( $\alpha 7 n A C h R)$. expressed on macrophages. This study defines the vagus nerve fibers that transmit the efferent signal in this pathway, a motor arc of the inflammatory reflex. Mice and rats were subjected to electrical cervical vagus nerve stimulation or sham surgery. Cytokine levels in serum were measured in endotoxemic animals or in endotoxin-exposed blood samples. Evoked potentials were measured in the vagus nerve and a portion of the vagus nerve was anesthetized using local application of lidocaine before stimulation. The lowest threshold subdiaphragmatic fibers in the rat vagus nerve have conduction velocities consistent with that of myelinated B fibers. The stimulation current threshold for significant suppression of serum TNF levels was similar in mice and rats ( $\leq 500 \mu A$ ). Blockade of the fibers caudal to the site of vagus nerve stimulation impaired the inhibition of TNF release. A single suprathreshold pulse stimulation was sufficient to suppress TNF release in endotoxemia. These results indicate that single-pulse and unidirectional electrical activation of the cervical vagus nerve reduce TNF in endotoxemia.
\end{abstract}

Online address: www.bioelecmed.org

doi: 10.15424/bioelectronmed.2015.00006

\section{INTRODUCTION}

Bioelectronic medicine, the interdisciplinary field that brings together neurophysiology, molecular medicine and biomedical engineering, has a significant potential to revolutionize treatment of inflammation and other diseases (1). The inflammatory reflex, a neural circuit that regulates inflammation, includes a sensory arc through the vagus nerve, and an efferent arc termed the cholinergic antiinflammatory pathway. Neurophysiological, genetic and molecular mechanistic studies of the inflammatory reflex have revealed that the integrity of the circuit is dependent upon action poten-

*PSO and YAL contributed equally to this work.

Address correspondence to Peder S Olofsson, Laboratory of Biomedical Science, The Feinstein Institute for Medical Research, 350 Community Drive, Manhasset, NY 11030. Phone: 516-234-1 127; Fax: 516-562-2356; E-mail: lolofsson@nshs.edu; or Yaakov A Levine, SetPoint Medical Corporation, 28368 Constellation Rd., Suite 300, Valencia, CA 91355. Phone: 661600-9536; E-mail: ylevine@setpointmedical.com.

Submitted February 12, 2015; Accepted for publication February 17, 2015; Published Online (www.bioelecmed.org) May 13, 2015.

\section{The Feinstein Institute for Medical Research Empowering Imagination. Pioneering Discovery.}

tials descending in the vagus nerve, splenic $\mathrm{T}$ cells that express choline acetyltransferase and red pulp macrophages that express alpha7 nicotinic acetylcholine receptors ( $\alpha 7 \mathrm{nAChR})$. Stimulation of the inflammatory reflex attenuates inflammatory disease in arthritis, colitis and other inflammatory diseases in animals (2-4) and in a clinical trial of rheumatoid arthritis patients (5). Ongoing clinical studies of implanted vagus nerve stimulators are focused on rheumatoid arthritis, inflammatory bowel disease and postoperative ileus $(6,7)$.

Despite the detailed mechanistic understanding of the inflammatory reflex, the neurophysiological identity of the efferent vagus nerve fibers in the motor arc of the inflammatory reflex have not been described. Prior neuroanatomical studies revealed a connection between 
A

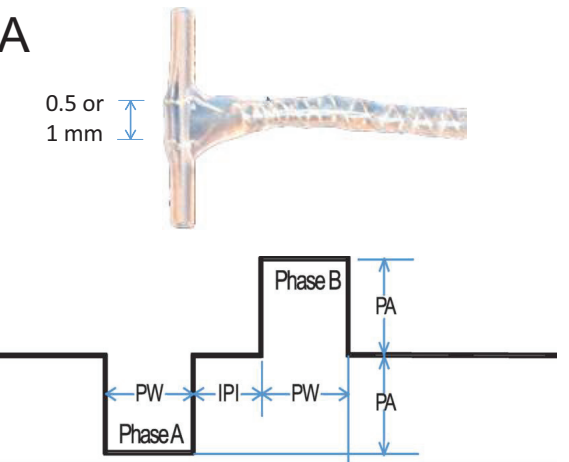

B

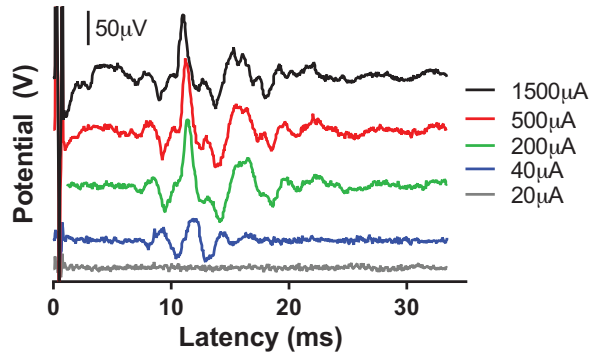

C

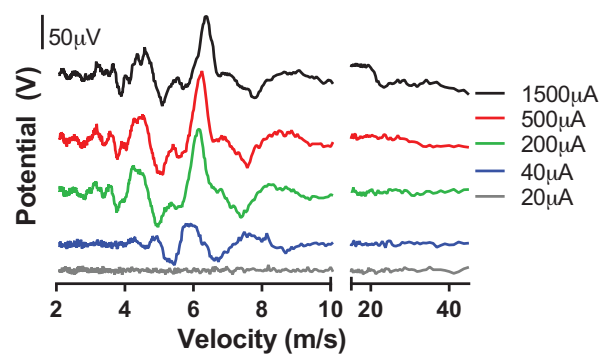

Figure 1. Vagus fibers in the motor arc of the inflammatory reflex. Electrical stimulation of the cervical vagus nerve was performed using a cuff-mounted bipolar stimulating electrode. (A) (†op) cuff electrode (bottom) pulse waveform. PA: pulse amplitude; PW: pulse width (200 to $250 \mu \mathrm{s})$; IPI: interpulse interval $(50 \mu \mathrm{s})$. (B,C) Evoked potentials were measured at the subdiaphragmatic vagus nerve in rats. Evoked potentials are plotted against (B) time and $(C)$ velocity $(n=4)$.

vagus nerve fibers and prevertebral ganglia, including the celiac and superior mesenteric ganglia, which is the origin of the adrenergic splenic nerve (8-11). It also has been established that action potentials arising in the splenic nerve as well as activation of acetylcholine-releasing $\mathrm{T}$ cells in spleen are required for the integrity of the inflammatory reflex and its ability to inhibit TNF release by splenic macrophages (12-14). Moreover, activation of isolated splenic neurons inhibits TNF production in isolated spleen preparations (15). The magnitude of the electrical stimulation sufficient to activate the inflammatory reflex is significantly less than the threshold required to induce bradycardia $(16,17)$, suggesting, though not proving, that the fibers that transmit the TNF inhibiting signal are distinct from the fibers that modulate heart rate. The cervical vagus nerve contains a large number of fibers, $>100,000$ in humans, predominantly unmyelinated $C$ fibers, and a minority of myelinated A and B fibers. Recent observations suggest that therapeutic effects of vagus nerve stimulation are associated with electrical activation of myelinated fibers (18). Accordingly, here we investigated stimulation thresholds and directionality requirements of electrical activation of the cervical vagus nerve to inhibit TNF production in the spleen during endotoxemia.

\section{MATERIALS AND METHODS}

This study was approved by The Feinstein Institute for Medical Research's Institutional Animal Care and Use Committee.

\section{Vagus Nerve Stimulation}

Male balb/C mice and Sprague Dawley rats were obtained from Taconic Farms Inc. (Hudson, NY, USA) and the studies were conducted at The Feinstein Institute for Medical Research (Manhasset, NY, USA). Animal care conformed to the Guide for the Care and Use of Laboratory Animals (19), and the applicable standard operating procedures of The Feinstein Institute for Medical Research. Animals were housed in a laboratory environment on a 12-h light-dark cycle at 19.5 to $24^{\circ} \mathrm{C}$ room temperature and relative humidity $30 \%-70 \%$ and had free access to food and water.

Mice and rats were anesthetized with intramuscular (IM) injection of ketamine (100 mg/kg) and xylazine (10 mg/kg). A ventral midline cervical incision was made between the mandible and sternum and subcutaneous tissue was dissected and retracted laterally. The mandibular salivary glands were bluntly separated and retracted laterally. The left carotid sheath was isolated between the sternomastoid and sternohyoid muscles. A custom-built bipolar cuff electrode (MicroProbes, Gaithersburg,
MD, USA) (Figure 1A) with a silasticcoated platinum-iridium wire lead and an internal diameter of $0.3 \mathrm{~mm}$ was secured about the outside of the entire carotid sheath containing the vagus nerve in mice and the isolated vagus nerve in rats. In some experiments, a cuff electrode was placed on both vagus nerves or on the right cervical vagus nerve. Sham-treated mice were handled similarly, but no electrical stimulation was applied.

Electrical pulses were delivered by a custom-built stimulator (SetPoint Medical Inc., Valencia, CA, USA) as 0 to 2,500 $\mu$ A charge-balanced biphasic square pulses with 200-250 $\mu$ s pulse width at $10 \mathrm{~Hz}$ for $60 \mathrm{~s}$, unless otherwise specified. In some experiments, $1.5 \mu \mathrm{L}$ of lidocaine $20 \mathrm{mg} / \mathrm{mL}$ was applied on the nerve cranial and/or caudal to the cuff electrode for 3 to $5 \mathrm{~min}$ prior to stimulation.

\section{Rodent Endotoxemia}

Three hours after VNS, endotoxin (LPS from Escherichia coli, 0111:B4; Sigma-Aldrich, St. Louis, MO, USA) was injected intraperitoneally (IP) ( $1 \mathrm{mg} / \mathrm{kg}$ in rats; $5 \mathrm{mg} / \mathrm{kg}$ in mice). Rodents were euthanized 90 min later and serum was collected for determination of TNF concentration by ELISA (R\&D Systems, Minneaspolis, MN, USA). 

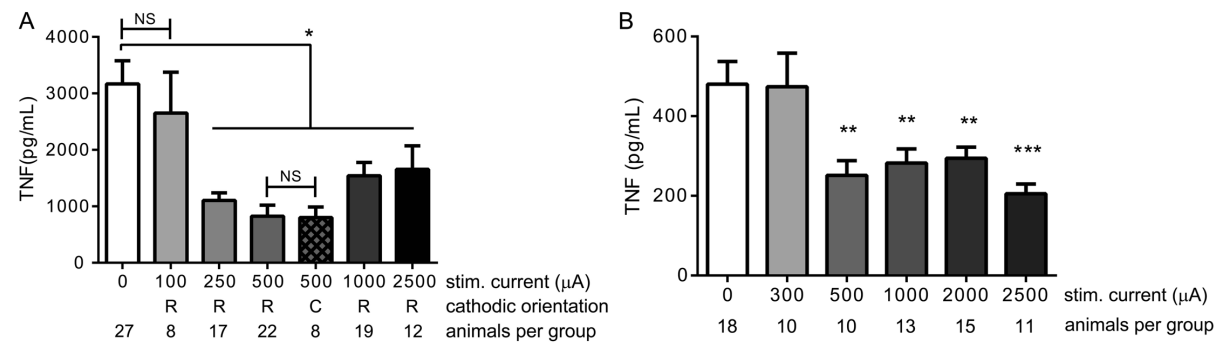

Figure 2. The stimulation-threshold current for the inflammatory reflex is similar in rats and mice. (A) Rats were subjected to endotoxemia after vagus nerve stimulation at increasing stimulation current intensities, and concentration of TNF measured in blood. $R$ and $C$ indicate rostral or caudal placement of the cathodic lead, respectively ( $n=8$ to 27 per group). (B) Mice were subjected to cervical vagus nerve stimulation using increasing stimulation current intensities and subjected to endotoxemia. The concentration of TNF measured in blood. Means \pm SEM are plotted. ${ }^{* *} p<0.01 ;{ }^{* * *} p<0.001$; NS, not significant.

\section{Measurement of Evoked Potentials in Rats}

A bipolar cuff electrode (MicroProbes) was placed on the left cervical vagus nerve, in isoflurane-anesthetized $(1.5 \%-3 \%)$ rats. The ventral trunk of the subdiaphragmatic vagus nerve was isolated through a midline abdominal incision and placed across a bipolar hook electrode (Plastics One Inc., Roanoke, VA, USA). The neural compound action potentials evoked by cervical VNS were sampled at $20 \mathrm{kHz}$, filtered (100 Hz to $10 \mathrm{kHz}, 60 \mathrm{~Hz}$ notch), and amplified $(50,000 \times)$ using an MP150 data acquisition system (Biopac Systems Inc., Goleta, CA, USA). The data was then averaged 60 times to increase signal-to-noise ratios.

\section{Statistical Analysis}

Differences between treatment groups were analyzed using analysis of variance (ANOVA) with Holm-Šidák post hoc test. Prism 6.0 (GraphPad software, San Diego, CA, USA) was used for statistical analyses. $p<0.05$ was considered significant.

\section{RESULTS}

\section{The Threshold of Electrical Cervical Vagus Nerve Stimulation for Inhibition of TNF in Endotoxemia Is Similar in Mice and Rats}

Electrical stimulation of the cervical vagus nerve (VNS) in Sprague Dawley rats with a charge-balanced, biphasic pulse (20) delivered through a bipolar stimulating electrode (see Figure 1A) elicited evoked potentials recorded in the subdiaphragmatic vagus nerve; the observed stimulation current threshold is $265 \pm 139 \mu \mathrm{A}$ (Figure 1B). The subdiaphragmatic fibers in the rat vagus nerve have conduction velocities of 4 to $9 \mathrm{~m} / \mathrm{s}$, consistent with activation of large myelinated, preganglionic B fibers (Figure 1C).

VNS currents at or above $250 \mu \mathrm{A}$ significantly inhibited serum TNF levels during endotoxemia in rats, while VNS at $\leq 100 \mu \mathrm{A}$ had no significant effect (Figure 2A). Application of vagus nerve stimulation using either caudal or cranial cathodic polarity significantly inhibited serum TNF levels (see Figure 2A). VNS also reduced TNF production in rat blood collected after nerve stimulation and exposed to endotoxin ex vivo (data not shown). Vagus nerve stimulation with $500 \mu \mathrm{A}$ in mice also significantly decreased endotoxin-induced serum TNF (Figure 2B). Together, these data indicate that a 1-min stimulation of $250-500 \mu \mathrm{A}$ is sufficient to activate the inflammatory reflex in rats and mice.

\section{Unidirectional Efferent Vagus Nerve Signals Are Sufficient to Suppress TNF in Endotoxemia}

Electrical stimulation of a nerve bundle can lead to activation of both afferent and efferent signals. To study the direc- tionality of vagus nerve signals required for inhibiting inflammatory response, we blocked efferent or afferent signals by application of a local anesthetic. Efferent, stimulation-evoked potentials recorded in the cervical vagus nerve were inhibited by lidocaine applied to the vagus nerve between the stimulation and recording sites (Figure 3A). Afferent vagus nerve blockade by lidocaine application cranial to the stimulation site did not abolish the reduction of TNF release mediated by cervical vagus nerve stimulation at the suprathreshold level of $1,000 \mu \mathrm{A}$ (Figure 3B). This is consistent with prior data that unidirectional efferent vagus nerve signaling inhibits spleen TNF production $(21,22)$. Simultaneous caudal and cranial lidocaine blockade significantly impaired the integrity of the inflammatory reflex, as we observed no suppression of endotoxininduced TNF levels by vagus nerve stimulation; unilateral lidocaine application without electrical stimulation also failed to inhibit serum TNF levels in endotoxemia (see Figure 3B). These findings indicate that activation of efferent, myelinated fibers in the motor arc of the inflammatory reflex are sufficient to reduce TNF release in endotoxemia.

These results do not exclude the possibility that afferent vagus nerve signals can modulate other pathways that regulate the cytokine response to endotoxin. Accordingly, we next blocked the efferent vagus nerve signals by applying anesthesia caudal to the stimulation cuff. Afferent vagus nerve stimulation under these experimental conditions also significantly reduced TNF release (Figure 3C), despite blockade of the efferent portion of the vagus nerve relative to the site of stimulation. Inhibition of TNF release in these experiments was not significantly different between caudal and cranial vagus nerve anesthesia. These data reveal additional TNF-inhibiting pathways mediated by afferent, or incoming signals, in the vagus nerve that can, in turn, activate descending, antiinflammatory mechanisms that are independent of the complete inflammatory reflex. 

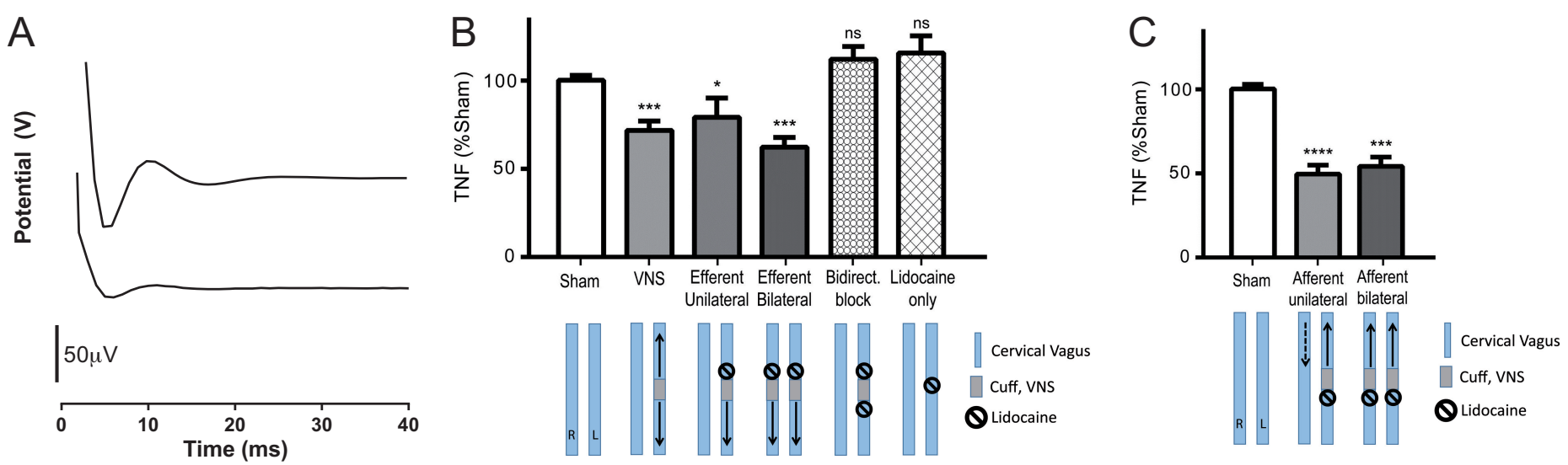

Figure 3. Unidirectional signals in the vagus nerve are sufficient to suppress TNF in endotoxemia. (A) Evoked potentials were recorded in the cervical vagus nerve in mice before (upper tracing) and after application of lidocaine caudal to the cuff-mounted stimulation electrodes (lower tracing). (B) Endotoxemia-induced blood levels of TNF were measured after vagus nerve stimulation in the presence of a lidocaineinduced block of the afferent vagus nerve in mice ( $n=7$ to 25 per group). (C) Endotoxemia-induced blood levels of TNF were measured after vagus nerve stimulation in the presence of a lidocaine-induced block of the efferent vagus nerve in mice ( $n=7$ to 25 per group). Mean \pm SEM TNF levels are plotted as percent of sham. ${ }^{*} p<0.05,{ }^{* * *} p<0.001,{ }^{* * *} p<0.0001$. VNS: vagus nerve stimulation at $1,000 \mu A$, 200 to $250 \mu$ s pulse width at $10 \mathrm{~Hz}$ for $60 \mathrm{~s}$.

We next addressed the response of the reflex circuit to repetitive stimulation. Accordingly, the cervical vagus nerve in anesthetized mice was stimulated repetitively from a single pulse, to up to 3,000 pulses. We observed that application of even a single-pulse stimulation reduced serum TNF levels (Figure 4). Addition of repetitive pulses failed to significantly enhance the inhibition of TNF, indicating that even a single stimulating pulse is sufficient to activate the inflammatory reflex.

\section{DISCUSSION}

These results indicate that single-pulse and unidirectional electrical activation of the cervical vagus nerve reduces TNF in endotoxemia.

As defined here and previously, the motor arc of the inflammatory reflex comprises action potentials in the vagus nerve that travel to the celiac ganglia to activate the adrenergic splenic nerve. The isolated motor arc is termed "the cholinergic antiinflammatory pathway," because neurotransmitter release from the splenic nerve triggers choline acetyltransferase ${ }^{+} \mathrm{T}$ cells in the spleen to release acetylcholine (23) which inhibits TNF release by activating $\alpha 7 \mathrm{nAChR}$ on monocytes and macrophages in the spleen (12,24-26). Multiple studies have confirmed and extended the original observations that signals in the vagus nerve reduce cytokine release in various inflammatory conditions by activating this efferent pathway that functionally connects the vagus nerve with immune cells in the spleen (3,8-11,21,27-29). Until now, the functional anatomy of the efferent vagus nerve pathway in the inflammatory reflex had been partially addressed, primarily in experiments of surgically dividing the nerve and assessing the effects on TNF release. In the present study, electrical propagation of vagus nerve signals was temporarily disabled by application of a local anesthetic. This approach offers the advantage of controlling the experimental conditions without permanently disrupting normal vagus neurophysiology. In agreement with previous findings, the present study shows that electrical stimulation of the efferent arc of the inflammatory reflex is sufficient to reduce TNF release in endotoxemia (21). The conduction velocity of the evoked potentials in the subdiaphragmatic vagus is consistent with the notion that large myelinated vagus nerve $B$ fibers propagate the signals in this efferent arc of the inflammatory re- flex. This observation does not, however, exclude the existence of other reflexively activated efferent nerves that regulate cytokine production and release.

Anesthetizing the vagus nerve caudal to the stimulation electrodes did not abol-

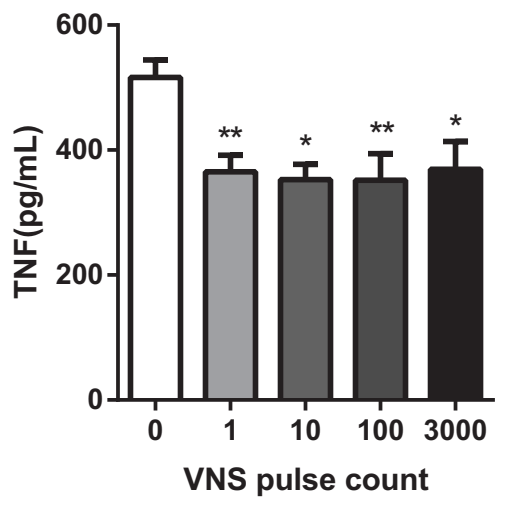

Figure 4. Single-pulse vagus nerve stimulation significantly reduced TNF. Rodents were subjected to 0 to 3,000 pulses of electrical vagus nerve stimulation and then rested for $3 \mathrm{~h}$ before induction of endotoxemia. TNF levels were measured in blood 90 min after intraperitoneal injection of endotoxin ( $n=8$ to 32 per group). Means \pm SEM are plotted. ${ }^{*} p<$ $0.05,{ }^{* *} p<0.01$ 
ish the effect of cervical vagus nerve stimulation on inhibition of TNF release in mice. This observation supports the presence of other antiinflammatory pathways from the central nervous system (CNS) that can inhibit cytokine release independently of the prototypical inflammatory reflex (reviewed in [30,31]). Such putative mechanisms include both signaling via the sympathetic chain to the splenic nerve and spleen and also activation of the hypothalamic-pituitary-adrenal axis to release glucocorticoids that, in turn, inhibit serum TNF (9,31-33). As we and others have discussed elsewhere, the inflammatory reflex is a prototypical cytokineregulating circuit, but not an exclusive one, and a number of neural reflexes have now been identified that can regulate inflammation and immunity $(4,34)$.

Other neural pathways that regulate immune cell activity and cytokine release includes vagus nerve-dependent inhibition of intestinal inflammation, which is independent of splenic signaling and, therefore, independent of the inflammatory reflex (35). Entry of pathogenic $\mathrm{T}$ cells across the blood brain barrier into the CNS is modulated by adrenergic efferent signals activated in response to proprioceptive signals originating in the lumbar spinal cord $(36,37)$. Sensory nerves in skin can be activated directly by bacterial products (38) and nociceptive neurons also can promote skin inflammation (39). Signals arising in adrenergic nerves are known to have important effects on immune cell activity $(40,41)$ and electric stimulation of the sciatic nerve regulates adrenal catecholamine-production via the vagus nerve (42). Together with other findings, these studies reveal that specific neural circuits regulate activation of immunity. As with other reflexes, which do not function in isolation, immune-regulating neural reflex pathways are not mutually exclusive. Rather, neural reflexes are integrated to maintain immunological homeostasis under a wide range of invasive and injurious threat.

Another major finding in this study is that a single electrical pulse applied to the vagus nerve significantly reduced serum
TNF levels. The single-pulse experiments were performed in mice at a supra-threshold current of $750 \mu \mathrm{A}$, corresponding to a total cathodal charge delivery of $150 \mathrm{pC}$. This discovery is important, because it suggests that a very short activation of implanted vagus nerve stimulators may be sufficient to have a treatment effect on inflammatory disease. Limited and ultralow duty cycle "on" time of an implanted stimulator will likely not only reduce unwanted side effects, but the low energy requirement will demand less stimulator battery power and may allow for new miniaturized form factors and longer intervals between device servicing. Furthermore, the vagus nerve stimulation threshold current for inhibition of TNF release in our experiments was 250-500 $\mu \mathrm{A}$ in mice and rats. The current requirements reported here at $10 \mathrm{~Hz}$ are significantly below the levels that previously have been described to reduce heart rate in rodents $(13,16,17)$. Together, these findings suggest that the motor arc of the inflammatory reflex is transmitted by motor fibers that are distinct from those that regulate heart rate.

\section{CONCLUSION}

Vagus nerve stimulation is emerging as a potential treatment for human inflammatory diseases $(5,6,43,44)$. The growing understanding of the components and signals that constitute the inflammatory reflex is driving the development of a potentially revolutionary line of novel therapeutic opportunities. This study shows that a single-pulse electrical activation of the vagus nerve is sufficient to reduce cytokine release, and that efferent and afferent vagus nerve signals have a separate capacity to regulate immunity. The implications of fiber specificity and the potential to target afferent and efferent pathways separately should inform development of future neuromodulation techniques to treat disease.

\section{ACKNOWLEDGMENTS}

We thank Warren M Grill for his insightful comments. This study was funded by SetPoint Medical Corporation, by the National Institute of General Medical Sciences (R01GM057226 to KJ Tracey and R01GM089807 to VA Pavlov) and by Svenska Läkaresällskapet.

\section{DISCLOSURE}

YA Levine, A Caravaca, and M Faltys are full time employees of SetPoint Medical Corporation.

\section{REFERENCES}

1. Famm K, Litt B, Tracey KJ, Boyden ES, Slaoui M. (2013) Drug discovery: a jump-start for electroceuticals. Nature. 496:159-61.

2. Olofsson PS, Rosas-Ballina M, Levine YA, Tracey KJ. (2012) Rethinking inflammation: Neural circuits in the regulation of immunity. Immunol. Rev. 248:188-204.

3. Andersson U, Tracey KJ. (2012) Reflex principles of immunological homeostasis. Annu. Rev. Immunol. 30:313-35.

4. Pavlov VA, Tracey KJ. (2012) The vagus nerve and the inflammatory reflex-linking immunity and metabolism. Nat. Rev. Endocrinol. 8:743-54.

5. Koopman FA, et al. (2012) Pilot study of stimulation of the cholinergic anti-inflammatory pathway with an implantable vagus nerve stimulation device in patients with rheumatoid arthritis [abstract]. Arthritis Rheum. 64:S195.

6. Zitnik RJ. (2011) Treatment of chronic inflammatory diseases with implantable medical devices. Cleve. Clin. J. Med. 78 Suppl 1:S30-4.

7. van Bree SH, et al. (2012) New therapeutic strategies for postoperative ileus. Nat. Rev. Gastroenterol. Hepatol. 9:675-83.

8. Berthoud HR, Powley TL. (1993) Characterization of vagal innervation to the rat celiac, suprarenal and mesenteric ganglia. J. Auton. Nerv. Syst. 42:153-69.

9. Berthoud HR, Powley TL. (1996) Interaction between parasympathetic and sympathetic nerves in prevertebral ganglia: morphological evidence for vagal efferent innervation of ganglion cells in the rat. Microsc. Res. Tech. 35:80-6.

10. Bellinger DL, Felten SY, Lorton D, Felten DL. (1989) Origin of noradrenergic innervation of the spleen in rats. Brain Behav. Immun. 3:291-311.

11. Nance DM, Burns J. (1989) Innervation of the spleen in the rat: evidence for absence of afferent innervation. Brain Behav. Immun 3:281-90.

12. Rosas-Ballina M, et al. (2008) Splenic nerve is required for cholinergic antiinflammatory pathway control of TNF in endotoxemia. Proc. Natl. Acad. Sci. U. S. A. 105:11008-13.

13. Huston JM, et al. (2006) Splenectomy inactivates the cholinergic antiinflammatory pathway during lethal endotoxemia and polymicrobial sepsis. J. Exp. Med. 203:1623-8.

14. Huston JM, et al. (2009) Cholinergic neural signals to the spleen down-regulate leukocyte trafficking via CD11b. J. Immunol. 183:552-9. 
15. Kees MG, Pongratz G, Kees F, Scholmerich J, Straub RH. (2003) Via beta-adrenoceptors, stimulation of extrasplenic sympathetic nerve fibers inhibits lipopolysaccharide-induced TNF secretion in perfused rat spleen. J. Neuroimmunol. 145:77-85.

16. Huston JM, et al. (2007) Transcutaneous vagus nerve stimulation reduces serum high mobility group box 1 levels and improves survival in murine sepsis. Crit. Care Med. 35:2762-8.

17. Woodbury DM, Woodbury JW. (1990) Effects of vagal stimulation on experimentally induced seizures in rats. Epilepsia. 31 Suppl 2:S7-19.

18. Yoo PB, et al. (2013) High-resolution measurement of electrically-evoked vagus nerve activity in the anesthetized dog. J. Neural. Eng. 10:026003.

19. Committee for the Update of the Guide for the Care and Use of Laboratory Animals, Institute for Laboratory Animal Research, Division on Earth and Life Studies, National Research Council of the National Academies. (2011) Guide for the Care and Use of Laboratory Animals. 8th edition. Washington (DC): The National Academies Press.

20. Levine YA, et al. (2014) Neurostimulation of the cholinergic anti-inflammatory pathway ameliorates disease in rat collagen-induced arthritis. PLoS One. 9:e104530.

21. Borovikova LV, et al. (2000) Vagus nerve stimulation attenuates the systemic inflammatory response to endotoxin. Nature. 405:458-62.

22. Vida G, Pena G, Deitch EA, Ulloa L. (2011) alpha7-cholinergic receptor mediates vagal induction of splenic norepinephrine. J. Immunol. 186:4340-6.

23. Rosas-Ballina M, et al. (2011) Acetylcholinesynthesizing $\mathrm{T}$ cells relay neural signals in a vagus nerve circuit. Science. 334:98-101.

24. Wang H, et al. (2003) Nicotinic acetylcholine receptor alpha7 subunit is an essential regulator of inflammation. Nature. 421:384-8.

25. Olofsson PS, et al. (2012) alpha7 nicotinic acetylcholine receptor (alpha7nAChR) expression in bone marrow-derived non-T cells is required for the inflammatory reflex. Mol. Med. 18:539-43.

26. Tracey KJ. (2002) The inflammatory reflex. Nature. 420:853-9.

27. Borovikova LV, et al. (2000) Role of vagus nerve signaling in CNI-1493-mediated suppression of acute inflammation. Auton. Neurosci. 85:141-7.

28. Rosas-Ballina M, et al. (2014) Xanomeline suppresses excessive pro-inflammatory cytokine responses through neural signal-mediated pathways and improves survival in lethal inflammation. Brain Behav. Immun. 44:19-27.

29. Mirakaj V, Dalli J, Granja T, Rosenberger P, Serhan CN. (2014) Vagus nerve controls resolution and pro-resolving mediators of inflammation. J. Exp. Med. 211:1037-48.

30. Andersson U, Tracey KJ. (2012) Neural reflexes in inflammation and immunity. J. Exp. Med. 209:1057-68.

31. Pavlov VA, Wang H, Czura CJ, Friedman SG, Tracey KJ. (2003) The cholinergic anti-inflammatory pathway: a missing link in neuroimmunomodulation. Mol. Med. 9:125-34.

32. Thayer JF, Sternberg E. (2006) Beyond heart rate variability: vagal regulation of allostatic systems. Ann. N. Y. Acad. Sci. 1088:361-72.

33. Pavlov VA, et al. (2009) Brain acetylcholinesterase activity controls systemic cytokine levels through the cholinergic anti-inflammatory pathway. Brain Behav. Immun. 23:41-5.

34. Sundman E, Olofsson PS. (2014) Neural control of the immune system. Adv. Physiol. Educ. 38:135-9.

35. Matteoli G, et al. (2013) A distinct vagal antiinflammatory pathway modulates intestinal muscularis resident macrophages independent of the spleen. Gut. 63:938-48.

36. Arima Y, et al. (2012) Regional neural activation defines a gateway for autoreactive T cells to cross the blood-brain barrier. Cell. 148:447-57.

37. Tracey KJ. (2012) Immune cells exploit a neural circuit to enter the CNS. Cell. 148:392-4.

38. Chiu IM, et al. (2013) Bacteria activate sensory neurons that modulate pain and inflammation. Nature. 501:52-7.

39. Riol-Blanco L, et al. (2014) Nociceptive sensory neurons drive interleukin-23-mediated psoriasiform skin inflammation. Nature. 510:157-61.

40. Elenkov IJ. (2008) Neurohormonal-cytokine interactions: implications for inflammation, common human diseases and well-being. Neurochem. Int. 52:40-51.

41. Elenkov IJ, Wilder RL, Chrousos GP, Vizi ES. (2000) The sympathetic nerve-an integrative interface between two supersystems: the brain and the immune system. Pharmacol. Rev. 52:595-638.

42. Torres-Rosas R, et al. (2014) Dopamine mediates vagal modulation of the immune system by electroacupuncture. Nat. Med. 20:291-5.

43. Koopman FA, et al. (2011) Restoring the balance of the autonomic nervous system as an innovative approach to the treatment of rheumatoid arthritis. Mol. Med 17:937-48.

44. Olofsson PS. (2014) A stimulating concept: bioelectronic medicine in inflammatory disease. Bioelectron. Med. 1:30-3.

Cite this article as: Olofsson PS, et al. (2015) Singlepulse and unidirectional electrical activation of the cervical vagus nerve reduces tumor necrosis factor in endotoxemia. Bioelectron. Med. 2:37-42. 